\title{
Thermal non-equilibrium of porous flow in a resting matrix applicable to melt migration: a parametric study
}

\author{
Laure Chevalier, Harro Schmeling \\ Institute of Geosciences, Goethe University, 60438 Frankfurt, Germany \\ 5 Correspondence to: Harro Schmeling (schmeling@geophysik.uni-frankfurt.de)
}

\begin{abstract}
Fluid flow through rock occurs in many geological settings on different scales, at different temperature conditions and with different flow velocities. Depending on these conditions the fluid will be in local thermal equilibrium with the host rock or not. To explore the physical parameters controlling thermal non-equilibrium the coupled heat equations for fluid and solid phases are formulated for a fluid migrating through a resting porous solid by Darcy flow. By non-dimensionalizing the

10 equations three non-dimensional numbers can be identified controlling thermal non-equilibrium: the Peclet number $P e$ describing the fluid velocity, the heat transfer number $A$ describing the local interfacial heat transfer from the fluid to the solid, and the porosity $\phi$. The equations are solved numerically for the fluid and solid temperature evolution for a simple 1D model setup with constant flow velocity. Three stages are observed: a transient stage followed by a stage with maximum nonequilibrium fluid to solid temperature difference, $\Delta T_{\max }$, and a stage approaching the steady state. A simplified time-

15 independent ordinary differential equation for depth-dependent $\left(T_{f}-T_{s}\right)$ is derived and analytically solved. From these solutions simple scaling laws of the form $\left(T_{f}-T_{S}\right)=f(P e, A, \phi, H)$, where $H$ is the non-dimensional model height, are derived. The solutions for $\Delta T_{\max }$ and the scaling laws are in good agreement with the numerical solutions. The parameter space $P e, A, \phi, H$ is systematically explored. In the $P e-A$ - parameter space three regimes can be identified: 1) at high $P e$ (>1) strong thermal non-equilibrium develops independently of $P e$ and $A ; 2)$ at low $P e(<1)$ and low $A(<1)$ non-equilibrium

20 decreases proportional to decreasing $P e ; 3)$ at low $P e(<1)$ and large $A(>1)$ non-equilbrium scales with $P e / A$ and thus becomes unimportant. The porosity $\phi$ has only a minor effect on thermal non-equilibrium. The time scales for reaching thermal nonequilibrium scale with the advective time-scale in the high $P e$-regime and with the interfacial diffusion time in the other two low $P e$ - regimes. Applying the results to natural magmatic systems such as mid-ocean ridges can be done by estimating appropriate orders of $P e$ and $A$. Plotting such typical ranges in the $P e-A$ regime diagram reveals that a) interstitial melt flow is

25 in thermal equilibrium, b) melt channelling as e.g. revealed by dunite channels may reach moderate thermal non-equilibrium, and c) the dyke regime is at full thermal non-equilibrium.
\end{abstract}


https://doi.org/10.5194/se-2021-149

Preprint. Discussion started: 21 December 2021

(c) Author(s) 2021. CC BY 4.0 License.

\section{(c) (i)}

\section{Introduction}

Fluid flow through rock occurs in many geological settings on different scales, at different temperature conditions and with different flow velocities. Depending on these conditions the fluid will be in local thermal equilibrium with the host rock or not. On small scale, e.g. grain scale, usually thermal equilibrium is valid. Examples include melt migration through a porous matrix in the asthenosphere or in crustal magmatic systems at super-solidus temperatures (e.g. McKenzie, 1984), groundwater or geothermal flows in sediments or cracked rocks (e.g. Verruijt, 1982; Furbish, 1997; Woods, 2015), or hydrothermal convection in the oceanic crust (e.g. Davis et al., 1999; Harris and Chapman, 2004; Becker and Davies, 2004). On a somewhat larger scale local thermal equilibrium may not always be reached. Examples of such flows include melt migration in the mantle or crust at temperatures close to or slightly below the solidus where melt may be focused and migrates through systems of veins or channels (Kelemen et al., 1995; Spiegelman et al., 2001). Within the upper oceanic crust also water may migrate through systems of vents or channels (Wilcock and Fisher, 2004). At even larger scales and at sub-solidus conditions magma rapidly flows through propagating dykes or volcanic conduits (e.g. Lister and Kerr, 1991; Rubin, 1995; Rivalta et al., 2015) and is locally at non-equilibrium with the host rock.

Heat transport associated with most of such flow scenarios is usually described by either assuming thermal equilibrium between the fluid and solid in case of slow flow velocity (e.g. McKenzie 1984) or, for more rapid flows such melts moving in dykes through a cold elastic or visco-elasto-plastic ambient rock, by assuming the fluids as isothermal (e.g. Maccaferri et al., 2011; Keller et al., 2013). However, on local scale of channel or dyke width thermal interaction between rising hot magma and cold host rock is important and may lead to effects such as melting of the host rock and freezing of the magma with important consequences for dyke propagation and the maximum ascent height (e.g. Bruce and Huppert, 1990; Lister and Kerr, 1991; Rubin, 1995). Clearly, in such rapid fluid flow scenarios melt is not in thermal equilibrium with the ambient rock.

Thus, there exists a transitional regime, which, for example, may be associated with melt focusing into pathways where flow is faster and thermal equilibrium might not be valid anymore. In such a scenario it might be possible that channelized flow of melt might penetrate deeply into sub-solidus ambient rock, and thermal non-equilibrium delays freezing of the ascending melts and promotes initiation of further dyke-like pathways. Indeed, for mid-oceanic ridges compositional non-equilibrium has proven to be of great importance for understanding melt migration and transport evolution (Aharonov et al., 1995; Spiegelman et al., 2001). Thus, it appears plausible that in cases of sufficiently rapid fluid flow e.g. due to channeling or fracturing thermal non-equilibrium may also become important. Describing this non-equilibrium macroscopically, i.e. on a scale larger than the pores or channels, is the scope of this paper.

While the physics of thermal non-equilibrium in porous flow is well studied in more technical literature (e.g. Amiri and Vafai, 1994; Minkowycz et al., 1999; Nield and Bejan, 2006; de Lemos, 2016), so far it has attracted only little attention in the geoscience literature, but see Schmeling et al., (2018) and Roy (2020). The basic approach in all these studies is the decomposition of the heat equation for porous flow into two equations, one for the solid and one for the migrating fluid. The 
equation for the fluid and as a source in the equation for the solid. Usually, this heat exchange term is assumed proportional to the local temperature difference between fluid and solid (Minkowycz et al. 1999; Amiri and Vafai, 1994; de Lemos, 2016; Roy, 2020), although Schmeling et al. (2018) showed that in a more general formulation the heat exchange term depends on the complete thermal history of the moving fluid through the possibly also moving solid. Here we will follow the common assumption and use the local temperature difference formulation. While Schmeling et al. (2018) showed that the magnitude of thermal non-equilibrium essentially depends on the flow velocity, or more general, on the Peclet number, here we will more generally explore the parameter space.

While thermal non-equilibrium of an arbitrary porous flow system depends on many parameters, our approach is to reduce the complexity of the system and systematically explore the non-dimensional parameter space. It will be shown that only three non-dimensional parameters control thermal non-equilibrium in porous flow, namely the Peclet number, an interfacial heat exchange number, and the porosity. The non-dimensionalization allows application of the results to arbitrary magmatic or other systems. The aim is to derive scaling laws allowing easily to decide whether thermal equilibrium or non-equilibrium is to be expected and to estimate the maximum temperature difference between fluid and matrix. The results will be applied to an anastomosing melt ascent system typical for mid-oceanic ridges in a second paper (Chevalier and Schmeling, in prep).

\section{Governing equations and model setup}

\subsection{Heat conservation equations}

We consider a homogeneous two-phase matrix-fluid system with a porosity constant in space and time. We assume a constant fluid velocity. The two phases are incompressible, and we assume local thermal non-equilibrium conditions, i.e. the two phases exchange heat. We solve the equations for conservation of energy (de Lemos, 2016) in this system.

80 Conservation of energy of the fluid phase is given by:

$c_{p, f}\left(\frac{\partial\left(\phi \rho_{f} T_{f}\right)}{\partial t}+\nabla \cdot\left(\phi \rho_{f} v_{f} T_{f}\right)\right)=\nabla \cdot\left(\phi \lambda_{f} \nabla T_{f}\right)-Q_{f s}$

For the definition of all quantities, see Table 1. Equation (1) can be further developed into:

$c_{p, f}\left(T_{f} \frac{\partial\left(\phi \rho_{f}\right)}{\partial t}+\phi \rho_{f} \frac{\partial T_{f}}{\partial t}+T_{f} \nabla \cdot\left(\phi \rho_{f} v_{f}\right)+\phi \rho_{f} v_{f} \cdot \nabla T_{f}\right)=\nabla \cdot\left(\phi \lambda_{f} \nabla T_{f}\right)-Q_{f s}$

Mass conservation for the fluid phase is given by:

$85 \frac{\partial\left(\rho_{f} \phi\right)}{\partial t}+\nabla \cdot\left(\rho_{f} \phi v_{f}\right)=0$

Inserting (3) into (2), conservation of energy for the fluid phase resumes to:

$c_{p, f} \rho_{f} \phi\left(\frac{\partial T_{f}}{\partial t}+v_{f} \cdot \nabla T_{f}\right)=\nabla \cdot\left(\phi \lambda_{f} \nabla T_{f}\right)-Q_{f s}$

In a similar way, the conservation of energy of the solid phase is given by:

$c_{p, s} \rho_{s}(1-\phi)\left(\frac{\partial T_{s}}{\partial t}+v_{s} \cdot \nabla T_{s}\right)=\nabla \cdot\left((1-\phi) \lambda_{s} \nabla T_{s}\right)+Q_{f s}$

90 which, assuming that $v_{s}=0$, is further simplified: 
The term $Q_{f s}$ in the fluid and solid heat conservation equations is the interfacial heat exchange term between the two phases (fluid and solid). In general, it depends on the local thermal history of the two phases and the history of the heat exchange (Schmeling et al., 2018). In a simplification it can be written as a combination of the interfacial area density $S$, the interfacial boundary layer thickness $d m$, the effective thermal conductivity $\lambda_{\text {eff }}$ and the temperatures of the two phases:

$Q_{f s}=\frac{s \lambda_{e f f}}{d m}\left(T_{f}-T_{s}\right)$

In general, the term $d m$ is time dependent. Schmeling et al. (2018) however provide evidence that taking an appropriate constant value for $d m$ (depending on fluid velocity) gives a good approximation of $Q_{f s}$ and allows for a reasonable modeling of temperature evolution with time. In most of the following parametric study, we use this simplification for $d m$ by assuming it is constant with time. The influence of time-dependence is discussed in section 5.1.4.

\subsection{Scaling and non-dimensionalization}

Non-dimensionalization is useful for interpreting models involving a large number of parameters. It usually helps reducing the number of parameters, and identifies non-dimensional parameters that control the evolution of the system. We write the two energy conservation equations in a non-dimensional form, using

$T=\Delta T_{0} T^{\prime}, t=t_{0} t^{\prime}, v=v_{0} v^{\prime},(x, y, z)=L_{0}\left(x^{\prime}, y^{\prime}, z^{\prime}\right)$

where $\Delta T_{0}$ is the macroscopic scaling temperature difference of the system, i.e. the initial temperature difference between top and bottom, $v_{0}$ is the scaling fluid velocity, $x, y, z$ is a distance, $L_{0}$ is the scaling length chosen as channel width of the pores, and

$110 t_{0}=L_{0}^{2} /\left(\kappa_{0} A\right)$

is the scaling time, where

$A=S^{\prime} /\left(\phi_{0} d m^{\prime}\right)$.

is defined as the heat transfer number. Primed quantities are non-dimensional. The scaling time and $A$ can also be written as

$t_{0}=L_{0} d m /\left(c \kappa_{0}\right)$

115 and

$A=c L_{0} / d m=c / d m^{\prime}$

where $\mathrm{c}$ is a geometrical constant of the order 2 for channels or 4 for tubes. Thus, $t_{0}$ represents the local diffusion time on a length scale defined by the geometric mean of the channel width, $L_{0}$, and interfacial boundary layer thickness, $d m$. The nondimensional heat transfer number $A$ scales with the ratio of the pore dimension to the interfacial boundary layer thickness, or

120 directly with the inverse non-dimensional boundary layer thickness.

Besides, we consider that the fluid and solid phases have the same densities and thermal properties: 
$c_{p, f}=c_{p, s}=c_{p, 0}, \quad \rho_{f}=\rho_{s}=\rho_{0}, \quad \kappa_{f}=\kappa_{s}=\frac{\lambda_{e f f}}{c_{p, 0} \rho_{0}}=\kappa_{0}$

This assumption is discussed in section 5.1.3 .

From Eq. (4), (6), and (7) we get the non-dimensional energy conservation equations for the fluid and solid phases,

respectively:

$\frac{\partial T_{f^{\prime}}}{\partial t^{\prime}}+\frac{P e}{A} v^{\prime} \cdot \nabla T_{f}^{\prime}=\frac{1}{\phi A} \nabla \cdot\left(\phi \nabla T_{f}^{\prime}\right)-\frac{\phi_{0}}{\phi}\left(T_{f}^{\prime}-T_{s}^{\prime}\right)$

$\frac{\partial T_{S^{\prime}}}{\partial t^{\prime}}=\frac{1}{(1-\phi) A} \nabla \cdot\left((1-\phi) \nabla T_{s}{ }^{\prime}\right)+\frac{\phi_{0}}{(1-\phi)}\left(T_{f}{ }^{\prime}-T_{s}{ }^{\prime}\right)$

From these equations we notice that the thermal evolution of the two-phase system is controlled by three non-dimensional parameters : $P e, A$, and $\phi_{0}$ where

$130 P e=\frac{v_{0} L_{0}}{\kappa_{0}}$

is the Peclet number. This number has already proven to be of high significance for determining whether thermal nonequilibrium is present or not (Schmeling et al. 2018), and the highest Pe corresponds to the largest temperature difference between fluid and matrix. In the following we drop the primes keeping all equations non-dimensional, only if nondimensionality is to emphasized, primes will be used.

135 From Eq. (12) and (13) two other controlling parameters can be identified: The heat transfer number $A$ characterizes heat transfer efficiency at the fluid-matrix interface. The larger $A$, the easier it is to transfer heat as $A$ scales directly with the inverse non-dimensional boundary layer thickness. The last controlling parameter is $\phi_{0}$, the scaling porosity.

In the following we will consider only models in $1 \mathrm{D}$, with constant porosity $\phi=\phi_{0}$ and with constant velocity. The latter will be expressed in terms of Pe, thus we choose $v^{\prime}=1$. This simplifies equations (12) and (13) to

$140 \frac{\partial T_{f}}{\partial t}+\frac{P e}{A} \frac{\partial T_{f}}{\partial z}=\frac{1}{A} \frac{\partial^{2} T_{f}}{\partial z^{2}}-\left(T_{f}-T_{s}\right)$

and

$\frac{\partial T_{S}}{\partial t}=\frac{1}{A} \frac{\partial^{2} T_{S}}{\partial z^{2}}+\frac{\phi_{0}}{\left(1-\phi_{0}\right)}\left(T_{f}-T_{S}\right)$,

respectively.

\subsection{Model setup}

145 The fluid and solid heat conservation equations are solved in 1D. Other geometries could also be easily explored but are not considered here, since we focus on studying the relative control of the scaling parameters on thermal non-equilibrium evolution. At time $t<0$, both solid and liquid are at rest, in equilibrium. For both phases temperatures are set to 1 (nondimensional temperature difference) at $z=0$, and a constant flux condition $\partial T / \partial z=1 / H$ (non-dimensional) is imposed at $z$ $=H$. Both initial temperatures decrease linearly from 1 to 0 with $z$, therefore a constant temperature gradient of $1 / H$ is present 150 in both phases (see Fig. 1). 


\subsection{Numerical scheme}

Equations are solved with a MATLAB (MATLAB R2018b ) code using a finite difference scheme central in space for the conduction terms, upwind for the advection term, and explicit in time. The spatial resolution is $d z=H / 10000$, and the time resolution was varied depending on the studied case.

\section{Numerical model results}

First some exemplary numerical results are shown in Fig. 2 to understand the physics and the typical behavior.

\subsection{Evolution of temperatures and thermal non-equilibrium with time}

Figure 2a and $\mathrm{b}$ represent $T_{f}$ and $T_{s}$ as functions of $z$ at different times for two different models. In both models, $P e=1, A=1$, $\phi=0.1$. However, while in the first model (Fig. 2a) $H=10$, in the second one (Fig. 2b) $H=100$. Figure $2 \mathrm{c}$ shows the evolution

160 of $T_{f}$ and $T_{s}$ with time at the top of the domain, for the same model as in Figure $2 \mathrm{~b}$. Figure $2 \mathrm{~d}$ represents the evolution of ( $T_{f}$ $T_{s}$ ) at different distances $z$ in model 2 (Fig. 2b).

At each depth of the system, the fluid and solid temperatures, as well as the temperature difference, appear to evolve following three stages:

Stage 1: During this transient stage the fluid temperature increases faster than the solid temperature (Fig. 2a,b,c,e), and the

165 temperature difference (Fig. 2d,f) increases. During this stage, the fluid temperature increases rapidly at first, then temperature increase slows down. As for the solid temperature, it first increases slowly, then faster and faster. At $t=0$, the fluid velocity is suddenly set to non-zero, thus the fluid temperature starts to deviate from equilibrium and increases in agreement with these new conditions. If the solid temperature were maintained constant with time, the fluid temperature would probably reach a steady-state profile, depending on boundary conditions, fluid velocity and solid temperature. As the fluid temperature increases

170 however, the liquid-solid temperature difference, thus the heat transfer term increases too, making the solid temperature to increase also faster and faster.

Stage 2: The fluid and the solid temperatures increase at similar rates, constant with time (Fig. 2c), the temperature difference remains stable (Fig. 2d). Solid-fluid heat transfer is maximum here.

Stage 3: As the fluid temperature rises close to the $T_{f}$ value at the bottom, its increase slows down, and heat transfer, thus

175 temperature difference, decreases. In model 1 (Fig. 2a), steady state is reached while the fluid and solid temperatures are still far from 1. This is due to the influence of boundary conditions, as the heat transferred from the fluid phase to the solid phase is compensated by the solid phase loss of heat at the top of the domain. In model 2 (Fig. 2b), boundary conditions are applied farther away from the bottom, therefore allowing for a higher increase of temperatures when reaching the steady state.

At each $z$ we observe that the temperature difference first increases rapidly to reach a maximum after a short time, here after $t$ $180=2$ (Fig. 2f). The resulting amplitude of the temperature difference is identical at the different z-positions. Then it stays constant at this maximum value, and finally decreases (Fig. 2d), as the fluid and solid temperatures experience the different 
stages. The higher in the model, the longer the temperature difference remains at maximum. This absolute maximum temperature difference in space and time does not depend on boundary conditions (see also section 5.1.2 where the influence of boundary conditions is discussed), nor on the z-position and therefore looks to be an interesting observable. It could indeed be useful for getting a first order estimate of thermal non-equilibrium conditions and possible temperature difference in a magmatic system. In the following sections we study how this maximum temperature difference evolves when varying the three parameters $P e, A$ and $\phi$.

\subsection{Maximum temperature difference}

The maximum temperature difference of a model can be defined as the maximum value reached in space and time (c.f. Fig.

2d). A series of models has been carried out for different parameters Pe, A, and $1-\phi$ (= solid fraction), and $\Delta T_{\max }$ has been determined for each model (Fig. 3). Some first observations can be made :

- For high $P e$ and high $A, \Delta T_{\max }$ is proportionnal to Pe/A (Fig. 3a) as long as $\Delta T_{\max }$ is smaller than the absolutely possible maximum 1.

- For small Pe, values align on a linear trend, proportional to $P e$ and independent of $A$ (Fig. 3a).

- The value of $A$ determines two main regimes, one in which $\Delta T_{\max }$ is proportional to Pe/A when $A$ is high (Fig. $3 \mathrm{~b}$ ), and one in which $\Delta T_{\max }$ is proportional to $P e$ when $A$ is small (Fig. 3a and b).

- $\Delta T_{\max }$ is proportional to $(1-\phi)$ for $P e=A$ larger than 0.1 (Fig. 3c).

These observations suggest the existence of several domains in which scaling laws for $\Delta T_{\max }$ could be derived, based on the three scaling parameters. In the next section, we propose an analytical retrieval of $\Delta T_{\max }$ values to derive these scaling laws and confirm these observations.

\section{Scaling laws derived from analytical solution}

In this section a simplified analytical solution for the z-dependent temperature difference between fluid and solid will be derived. From this solutions the maximum temperature differences $\Delta T_{\max }$ can be retrieved and scaling laws will be derived.

\subsection{Analytical solution of the governing equations}

205 The subtraction of Eq. (16) from Eq. (15) gives the following non-dimensional 1D equation:

$\frac{\partial\left(T_{f}-T_{S}\right)}{\partial t}-\frac{1}{A} \frac{\partial^{2}\left(T_{f}-T_{S}\right)}{\partial z^{2}}+\frac{P e}{A} \frac{\partial T_{f}}{\partial z}+\frac{1}{1-\phi}\left(T_{f}-T_{S}\right)=0$

which is equivalent to:

$\frac{\partial\left(T_{f}-T_{S}\right)}{\partial t}-\frac{1}{A} \frac{\partial^{2}\left(T_{f}-T_{S}\right)}{\partial z^{2}}+\frac{P e}{A} \frac{\partial\left(T_{f}-T_{S}\right)}{\partial z}+\frac{1}{1-\phi}\left(T_{f}-T_{S}\right)=-\frac{P e}{A} \frac{\partial T_{S}}{\partial z}$

Remember that $\phi=\phi_{0}$ is assumed constant. We simplify the problem by considering the hypothetical case in which $\left(T_{f}-T_{S}\right)$ does not change with time, and, moreover, in which the thermal gradient in the solid phase is linear, with $\partial T_{s} / \partial z=\Delta T / H$. 
Although different from initial and steady-state stages described in the 1D models (section 3.1), this hypothetical case is quite similar to what can be observed at the very beginning of the second stage described in section 3.1 (c.f. Fig. 2 d,f). In this second stage, the evolution of $T_{f}$ and $T_{s}$ was indeed observed to be quite similar. Besides, at the end of stage 1 (section 3.1), $T_{s}$ remains close to initial conditions, therefore to a linear gradient of slope $\Delta T / H$ is justified. Since the maximum temperature difference between the two phases is observed starting from the end of stage 1 and during stage 2 (section 3.2), it does not seem unreasonable to consider this hypothetical case for retrieving the maximum temperature difference. Using these assumptions, Eq. (18) resumes to:

$\frac{1}{A} \frac{\partial^{2}\left(T_{f}-T_{S}\right)}{\partial z^{2}}-\frac{P e}{A} \frac{\partial\left(T_{f}-T_{S}\right)}{\partial z}-\frac{1}{1-\phi}\left(T_{f}-T_{S}\right)=-\frac{P e}{A} \frac{\Delta T}{H}$

Eq. (19) is a second order ordinary differential equation for $\left(T_{f}-T_{S}\right)$ whose solution can be analytically retrieved and is given in Eq. (20) to (22) (details on the equation analytical solving are given in the supplementary material).

$T_{f}-T_{s}=\alpha e^{r_{1} z}+\beta e^{r_{2} z}+(1-\phi) \frac{P e}{A} \frac{\Delta T}{H}$,

where $r_{1}$ and $r_{2}$ are the roots of the associated equation of Eq. (19)

$r_{1}=\frac{P e-\sqrt{P e^{2}+\frac{4 A}{1-\phi}}}{2}, \quad r_{2}=\frac{P e+\sqrt{P e^{2}+\frac{4 A}{1-\phi}}}{2}$,

$\alpha$ and $\beta$ are constrained by the boundary conditions for $\left(T_{f}-T_{s}\right)\left(\left(T_{f}-T_{s}\right)=0\right.$ at $z=0$ and $\frac{\partial\left(T_{f}-T_{s}\right)}{\partial z}=0$ at $\left.z=H\right)$

$\alpha=(1-\phi) \frac{P e}{A} \frac{\Delta T}{H} \frac{r_{2}}{r_{1} e^{\left(r_{1}-r_{2}\right) H}-r_{2}}, \quad \beta=(1-\phi) \frac{P e}{A} \frac{\Delta T}{H} \frac{r_{1}}{r_{2} e^{\left(r_{2}-r_{1}\right) H}-r_{1}} \quad$,

and the third term in Eq. (20) is a particular solution for Eq. (19).

\subsection{Comparison with numerical models}

From Eq. (20) the maximum value of the depth-dependent temperature difference $\left(T_{f}-T_{s}\right)$ can be determined. It is found that the maximum is always at $z=H$. This value will be denoted as $\Delta T_{\max }$. Using this value the ratio of the analytical (Eq. 20 ) to the numerically determined $\Delta T_{\max }$ has been calculated for all 123 models studied and is shown as a function of $P e$ in Fig. S1 in the supplementary material. No correlation with $P e$ is observed. For $91 \%$ of the models, these ratios lie within 0.99 and 1.02, and all but one models are between 0.98 and 1.05. This surprisingly good agreement is another justification of using the time-independent equation (19) to retrieve an analytical solution of an intrinsically time-dependent process as long as we are interested only in the maximum value of $\left(T_{f}-T_{s}\right)$. Other reasons for the observed differences between the analytical and numerical solutions include numerical errors when determining the particular times when maximum temperature differences are reached, especially for the models which are in the transient regime.

\subsection{Scaling laws for temperature differences at certain parameter limits}

The analytical solution for $\Delta T_{\max }$ fits very well with our model results and therefore looks to be ideal for getting a better understanding on the relative influences of the three controlling parameters described in section 2.2. The Peclet number is 
240 already known to be of great importance for thermal equilibrium/non-equilibrium conditions. Although less commented for non-equilibrium build up, the heat transfer number $A$ controls local heat transfer between the two phases. We noticed in section 3.2 that there exist a high $A$ and a low $A$ regime with different non-equilibrium behavior. Inspecting Eq. (20) we notice that a high $P e$ should favor higher temperature differences, while a high $A$ would rather favor thermal equilibrium. The porosity $\phi$, in the form of (1- $\phi)$, also influences thermal equilibrium conditions, as expected in section 3.2.

245 Eq. (20) is, however, complex, and the visibility on the relative importance of these controlling parameters and of the conditions for different possible regimes is limited. In this section, we study the evolution of $\left(T_{f}-T_{s}\right)$, i.e. also $\Delta T_{\max }$, in a few limiting cases. This enables us to better understand each parameter influence and to derive some scaling laws for different regimes.

\subsubsection{Limit $\mathrm{A} \rightarrow 0$}

Linearizing Eq. (20) with respect to $A$, around $A=0$, using the Taylor series in terms of $A$, we derive the following limit for $250\left(T_{f}-T_{S}\right)$ (see supplementary material for more details):

$T_{f}-T_{S}=\frac{\Delta T}{H}\left(z+\frac{1}{P e e^{P e H}}\left(1-e^{P e z}\right)\right)$

The limit for $\Delta T_{\max }$ is simply obtained by setting $z=H$. The limit (23) can also be retrieved by neglecting the heat transfer term (left hand third term) in Eq. (19), which becomes negligible when $A$ tends to 0, and solving the resulting equation. From Eq. (23) we notice that when $A$ tends to 0 the temperature difference between the two phases does not depend on $A$ anymore.

255 It should be noted that this limit is valid only as long as $P e$ remains finite in the limit $A \rightarrow 0$. Further details of the comparison of Eq. (23) to the full analytical solution are discussed in the supplementary material.

\subsubsection{Limit $A \rightarrow \infty$}

When $A$ tends towards infinity, Eq. (20) tends to the following limit:

$T_{f}-T_{S}=(1-\phi) \frac{P e}{A} \frac{\Delta T}{H}$

260 Using this limit, we find values in very good agreement with those predicted by Eq. (20) for A>1.See also Fig. S2b in the supplementary material.

\subsubsection{Limit Pe $\rightarrow 0$}

When $P e$ tends to 0, Eq. (20) tends to the following limit (see supplementary material):

$T_{f}-T_{S}=(1-\phi) \frac{P e}{A} \frac{\Delta T}{H}\left(1-\frac{e^{-\sqrt{\frac{A}{(1-\phi)}}^{Z}}}{1+e^{-\sqrt{\frac{A}{(1-\phi)}}^{2}}}-\frac{e^{\sqrt{\frac{A}{(1-\phi)}}^{Z}}}{1+e^{\sqrt{\frac{A}{(1-\phi)}}^{2}}}\right)$

265 This limit gives predictions for $\Delta T_{\max }$ in very good agreement with Eq. (20) for $P e<1$ (having $A=1$ and $\phi=0.1$ ) (see Fig. S2a in the supplementary material).

If in addition $A$ tends to zero, we get the following limit: 
$T_{f}-T_{s}=\operatorname{Pe} \Delta T z\left(1-\frac{z}{2 H}\right)$

which is in agreement with the observation of a proportional relationship between $P e$ and $\Delta T_{\max }$ for small $A$ and small $P e(\Delta T$ was non-dimensional and equal to 1 in all models). For more discussion, see supplementary material.

\subsubsection{Limit Pe $\rightarrow \infty$}

To obtain the limit of Eq. (20) for $P e \rightarrow \infty$, Eq. (20) can be linearized with respect to $4 \mathrm{~A} /\left((1-\phi) P e^{2}\right) \ll 1$. Applying the rule of L'Hospital Eq. (20) tends to the following limit (for arbitrary A):

$T_{f}-T_{S}=\frac{\Delta T}{H} z$

275 For details, see supplementary material. This limit is also the solution of Eq. (19) when neglecting the diffusive and heat transfer terms. As demonstrated in the supplementary material this limit predicts $\Delta T_{\max }$ values in very good agreement with Eq. (20) for $P e>100$.

\subsubsection{Limit $\Phi \rightarrow 1$}

In Eq. (20), the presence of $\phi$ is always in the form of $A /(1-\phi)$. Therefore $\phi$ tending to 1 has the same limit as $A$ tending towards infinity (Eq. 24). This limit approaches values predicted by Eq. (20) for high $\phi$ values (Fig.S2d in the supplementary material).

\subsubsection{Exploring the domains for the maximum temperature difference including all limits}

Before exploring the full parameter space we first give a short overview of expected parameter ranges in magmatic systems. In natural magmatic systems such as mid-ocean ridges, $P e$ is expected to evolve from very low values of order $10^{-5}$ to $10^{-3}$ in partially molten regions with distributed porous flow to higher values of order 1 or larger at depths where channels have merged, and further to very high values of order $10^{5}$ in dyke systems (Schmeling et al., 2018). As $A$ scales with the ratio of the channel width to the interfacial boundary layer thickness, $A$ would evolve proportionally with the width of melt pathways which may increase by 3 to 5 orders of magnitude as $3 \mathrm{D}$ grain junctions eventually merge to $1 \mathrm{D}$ dykes . Additionally, as $A$ is inversely proportional to the interfacial boundary layer thickness which increases with time, $A$ should evolve with time, from high values at melt flow onset to much smaller values in highly evolved systems. In Figure 6 of Schmeling et al. (2018) the time-dependent interfacial heat flow has been determined which roughly maps into $A$ if multiplied by $\frac{\phi}{(1-\phi)}$. This suggests $A$ values dropping from about 10 to $10^{-5}$ over the time scale until thermal equilibrium is reached.

As melt flow may occur at very small melt fractions (McKenzie, 2000; Landwehr et al., 2001), large $\phi$ - values are not expected in natural mantle magmatic systems, nor in dyke systems in the crust. Values of channel volume fraction generally remain below a few percent up to tens of percent (in dunite channels up to 10 - 20\%, Kelemen et al., 1997). (1- $\phi$ ) would therefore not vary over more than one order of magnitude, making the porosity a less influential parameter on thermal non-equilibrium build up in magmatic systems. Indeed, we explored the dependence of $\Delta T_{\max }$ on $\phi$, for a number of very different combinations of 
$P e$ and $A$ values. Within possible ranges of $P e$ between $10^{-10}$ to $10^{5}$ and $A$ between $10^{-6}$ and $10^{2}$ resulting variations of $\Delta T_{\max }$ with mantle realistic $\phi$ values $(0-20 \%)$ are only of about 0.01 if normalized by $\Delta T_{0}$.

Because of this relatively small influence of $\phi$ on $\Delta T_{\max }$, we here explore $\Delta T_{\max }$ variations using the analytical solution Eq. (20), in which $\Delta T_{\max }$ depends essentially on $P e$ and $A$, and the reference value for $\phi$ is chosen as 0.1 . In the resulting $P e-\mathrm{A}$ diagram three main regimes can be distinguished (Fig. 4):

- Regime 1: For high $P e$ values, $\left(T_{f}-T_{s}\right)$ tends to the relationship described in Eq. (27). The temperature difference increases with distance from the bottom $(\mathrm{z}=0)$ reaching $\Delta T_{\max }=1$ at $\mathrm{z}=1$. In the whole region the fluid temperature remains constant and at maximum 1 while the solid temperature increases linearly with $\mathrm{z}$ from 0 to 1 .

- Regime 2: For small $A$ values and $P e<1,\left(T_{f}-T_{s}\right)$ is also dependent on the distance from the bottom, and is proportional to $P e$, while $A$ - and $\phi$ - influences are negligible. In this domain, $\left(T_{f}-T_{s}\right)$ tends to the relationships presented in Eq. (25) and (26).

- Regime 3: For high $A$ - values and $P e / A<1,\left(T_{f}-T_{s}\right)$ tends to the relationship proposed in Eq. (24). In this domain, $\left(T_{f}-T_{s}\right)=\Delta T_{\max }$ is no more dependent on depth, but is proportional to (1- $\left.\phi\right), \mathrm{Pe} / \mathrm{A}$ and to the initial solid temperature gradient, which is identical to the solid temperature gradient at the top, However, numerical time-dependent solutions show that $\left(T_{f}-T_{s}\right)=\Delta T_{\max }$ is essentially independent of depth only during stage 1 and 2 (c.f. Section 3.1) and for sufficiently large $\mathrm{H}(>5)$. Later it becomes depth-dependent and smaller than $\Delta T_{\max }$ given by Eq. (24).

\section{Discussion}

\subsection{Limitations}

\subsubsection{Comments onm the analytic solution}

Although the assumptions used to get the analytic solution (Eq. 20) are very specific, they are reasonable considering the conditions in the models when $\Delta T_{\max }$ is reached, and it fits very well the numerical results. This is shown in Fig. 5 where for various combinations of $P e, A$ and $H$ the time-dependent temperature differences $\left(T_{f}-T_{S}\right)$ are shown as functions of depth together with the analytical solutions using Eq. (20). For all examples the position of the maximum temperature differences lies at $z=H$. A major simplification used in Eq. (19) was time-independence. Obviously, the resulting analytical solutions represent the stage 2 , which is quasi steady state in contrast to stage 1 when the temperature difference builds up, and stage 3 when the long-term behavior is approached. We emphasize that this analytical solution is a very good approximation of the depth-dependent temporal maximum temperature difference that can be reached in such porous systems.

\subsubsection{Initial conditions and boundary conditions at top}

325 The boundary conditions we chose at the top $(z=H)$ are suitable for cases with little temperature evolution (regime 2), but might be inappropriate for high temperature increases (high $P e$-regimes) (see section 4.3.6). In order to quantify the influence 
of this choice of boundary conditions on our results, we compared $\left(T_{f}-T_{S}\right)$ - profiles for three models, that belong to the three regimes identified in section 4.3.6, using three different boundary conditions at the top:

- Constant heat flux equal to initial flux in the solid and fluid phases (Neumann conditions). This was the boundary condition used in the models.

- Heat flux is set to 0 at the top (Neumann conditions).

- Both fluid and solid temperatures are set to 0 at the top (Dirichlet conditions).

Although top boundary conditions can strongly affect fluid and solid temperature profiles (Fig. S 3, in supplementary material), their influence on $\left(T_{f}-T_{s}\right)$ is negligible for regimes 1 and 3, except when getting close to the top of the domain. The choice of the boundary conditions has, however, a much stronger influence on the whole profile for regime 2 , where temperature evolution and steady state are controlled by diffusion (see section 5.2 below), and are therefore very sensitive to boundary conditions (see Fig. S3 in supplementary material). We summarize that the influence of boundary conditions on fluid and solid temperatures evolution depends mostly on the domain size $(H)$ and on the value of $P e$ : The larger these two parameters, the less important is the influence of boundary conditions within almost the whole model region.

340 As initial condition we used a linear temperature profile and initial equilibrium between solid and fluid. A non-linear initial temperature profile between $T_{f}=T_{s}=1$ at the bottom and $T_{f}=T_{s}=0$ at the top would have spatially varying temperature gradients with sections with gradients larger than those assumed in our model. As the temperature gradient strongly influences thermal non-equilibrium (see e.g. Eq. 20 which explicitly contains the temperature gradient $\frac{\Delta T}{H}$ ), the above results are expected to be different, and a stronger thermal non-equilibrium is expected in regions with higher gradients. Schmeling et al. (2018) used a step function with $T_{f}=T_{s}=1$ at $z=0$ and $T_{f}=T_{s}=0$ at $z>0$ as initial condition, i.e. an extremely non-linear profile near $z=0$. Assuming this initial temperature profile Figure 6 shows the temporal behavior of the temperature difference for selected parameter combinations, equal to the parameters used in Fig. 5. The analytical solutions for the time-independent case (Eq. 20) is also shown. As expected, at early stages the temperature differences are significantly larger than given by the analytical solutions by a factor 2 or more shortly after the onset of the evolution. At later stages (stage 2 or 3 ) the timedependent solutions approach or pass through the analytical solutions. Thus, we may state that the analytical solutions depicted in the regime diagram in Fig. 4 represent lower bounds of thermal non-equilibrium compared to settings with non-linear initial temperature profiles.

\subsubsection{Densities and thermal properties of the two phases}

While for simplicity we used equal physical properties for the fluid and solid, in many circumstances they might be significantly different. Equal properties are good approximations for magmatic systems where differences of density and thermal parameters are small (order of 10\%), whereas porous flows of water or gases through rocks or other technical settings may be characterized by larger differences. Allowing for different material properties adds four new parameters, namely the ratio of diffusivities, the ratio of densities, the ratio of heat capacities and a new effective thermal conductivity $\lambda_{\text {eff }}$ for the 
interface between the two phases with different properties. To evaluate how many new non-dimensional numbers are introduced we non-dimensionalize the equations assuming different material properties for the two phases. We use the fluid properties as scaling quantities and assume that they are independent of temperature, pressure and depth. Eq. (12) and (13) turn into:

$\frac{\partial T_{f^{\prime}}}{\partial t^{\prime}}+\frac{P e}{A} \frac{1}{\lambda_{e f f^{\prime}}} v^{\prime} \cdot \nabla T_{f}^{\prime}=\frac{1}{\phi A \lambda_{e f f^{\prime}}} \nabla \cdot\left(\phi \nabla T_{f}^{\prime}\right)-\frac{\phi_{0}}{\phi}\left(T_{f}{ }^{\prime}-T_{s}{ }^{\prime}\right)$

and

$\frac{\partial T_{S^{\prime}}}{\partial t^{\prime}}=\frac{1}{(1-\phi) A} \frac{\kappa_{s^{\prime}}}{\lambda_{e f f^{\prime}}} \nabla \cdot\left((1-\phi) \nabla T_{s}{ }^{\prime}\right)+\frac{\phi_{0}}{(1-\phi)} \frac{1}{\rho_{s^{\prime}} c_{p, s^{\prime}}}\left(T_{f}{ }^{\prime}-T_{s}{ }^{\prime}\right)$

Inspection of these equations shows that two more non-dimensional numbers are introduced: the ratio of diffusivities $\kappa_{s}{ }^{\prime}$, and the ratio of the products density and heat capacity, $\rho_{s}{ }^{\prime} c_{p, s}{ }^{\prime}$. The new effective conductivity for heat transfer, $\lambda_{e f f}{ }^{\prime}$, can be merged with $A$ to define a new heat transfer number $A \lambda_{\text {eff }}{ }^{\prime}$.

As equations (28) and (29) cannot be merged into one time-independent ordinary differential equation for $\left(T_{f}-T_{S}\right)$ as in section 4.1, we numerically tested some cases with $P e=1$ and $A \lambda_{\text {eff }}{ }^{\prime}=1$ in which the diffusivity ratios and the ratios of $\rho_{s}{ }^{\prime} c_{p, s}{ }^{\prime}$ were varied between 0.1 and 10 (see Fig. S4 in the supplementary material). The results show that for the fixed combination of $P e=1$ and $A \lambda_{e f f}^{\prime}=1$ the magnitude of thermal non-equilibrium remains in the same order of magnitude $O(0.1)$ as for equal properties (Fig. 5a). However, the time-dependence is significantly effected: For a high ratio of $\kappa_{s}^{\prime}=10$ (i.e. the solid is strongly conducting) the solid temperature profile remains close to the constant initial gradient, and the temperature difference rapidly converges to a steady state similar to the analytical solution depicted in Fig. $5 \mathrm{a}$. In contrast, for a low $\kappa_{s}^{\prime}=0.1$ the solid temperature departs more strongly from the initial linear gradient, and the solid - fluid temperature difference slowly drops with time on the long term. Varying the potential to store heat in the solid, i.e. $\rho_{s}{ }^{\prime} c_{p, s}{ }^{\prime}$, shows that a high value slows down the time-dependent variations, while a small value leads to rapid temporal variations of $\left(T_{f}-T_{s}\right)$ and some differences in the final steady states (Fig. S4 e and $\mathrm{f}$ in the supplementary material).

380 It is interesting to apply the results for different physical properties to a geologically relevant setting, namely water flowing through sedimentary rocks. Given that the high heat capacity of water is about three times larger than that of rock, and the density is almost three times less, the product $\rho_{s}{ }^{\prime} c_{p, s}{ }^{\prime}$ is about 0.78 , i.e. of order 1 . However, the thermal diffusivity of water is significantly smaller than that of rock, typically by a factor 16 , i.e. $\kappa_{s}{ }^{\prime}$ is about 16 . We tested a few cases with Peclet numbers and $A$ (assuming for simplicity $\lambda_{e f f}{ }^{\prime}=1$ ) equal to the cases depicted in Fig. 5. The time dependent profiles behave 385 similarly to those in Fig. 5, with very similar maxima of the temperature differences (red dashed curves in Fig. 5) relevant for stage 2 . The only important difference is that the water-sedimentary rock case more rapidly approaches the late steady states of stage 3 and these stages are closer to the maximum red-dashed curves. The full set of results is shown in the supplementary material in Fig. S5. These results suggest that the absolute values of maximum thermal non-equilibrium temperature differences shown in the regime diagram Fig. 4 are also applicable to a water-sedimentary rock system. 
https://doi.org/10.5194/se-2021-149

Preprint. Discussion started: 21 December 2021

(c) Author(s) 2021. CC BY 4.0 License.

(c) (i)

390 applying a convolution integration over the past history of the thermal evolution. As this is numerically expensive, a first order step in this direction is to consider a spatially constant but time-dependent $A$ using boundary layer theory. To test this idea we replace the parameter $A$ used in the non-dimensionalization (Eq. 9) by a constant $A_{0}$ and use a microscopic thermal boundary layer thickening proportional to the square root of time, $d m=c_{t h} \sqrt{\kappa_{0} t}$, where $c_{t h}$ is a constant of order 2.32 for a cooling half-space (Turcotte and Schubert, 2014). Applying our non-dimensionalization a time-dependent $A$ can be defined as $A=\frac{c \sqrt{A_{0}}}{c_{t h} \sqrt{t}}$

400 From Eq. (30) it is clear that at the onset of our experiments $A$ is very large and then drops as time proceeds. Thus, from the regime diagram (Fig. 4) it is expected that in the early stage a large $A$ will lead to small temperature differences, while during later stages $A$ decreases, i.e. heat transfer between solid and fluid decreases, and thermal non-equilibrium might be built up. To include a varying $A\left(t^{\prime}\right)$ according to Eq. (30) the non-dimensional heat equations for the fluid and solid, Eq. (12) and (13), respectively, are modified by replacing all occurrences of $A$ by $A_{0}$ and multiplying the heat exchange terms (last terms) in both equations with $A\left(t^{\prime}\right) / A_{0}$. We carried out a few tests for similar parameters as chosen in Fig. 5, namely $P e=$ $0.01,1,100, A_{0}=0.01,1,100$. The results are shown in Fig. S6 in the supplementary material. It should be noted that from Eq. (30) a small $A_{0}=0.01$ corresponds to a time-tependent $A\left(t^{\prime}\right)$ dropping from order 1 to 0.01 , and a large $A_{0}=100$ corresponds to a time-dependent $A\left(t^{\prime}\right)$ dropping from order 100 to 1 during the time period of interest. Comparing the timedependent $A$-models with the constant $A$-models shows: 1$)$ the temporal behavior of $\left(T_{f}-T_{S}\right)$ is stronger for the variable $A$ models, and 2) the late stages are characterized by significantly larger $\left(T_{f}-T_{s}\right)$ - differences than the analytical maximum curves (dashed red) in Fig. 5. Only models with small $A_{0}$ and large $P e$ remain comparable to models with constant $A_{0}$. Thus, we may state here that the thermal non-equilibrium temperature differences given in the regime diagram (Fig. 4) provide lower bounds for systems in which the heat transfer parameter is allowed to vary with time. Qualitatively, a time-dependent $A$ shifts the boundary between large and small temperature differences in the regime diagram to the right and downwards. More work

415 needs to be done to fully explore the regime diagram for time-dependent $A$ and compare such models with the fully consistent solutions of Schmeling et al. (2018).

\subsubsection{Spatially constant parameters}

Here we assumed spatially constant parameters $\phi, P e, A$. In reality, percolating melt may focus into channels which subsequently merge forming anastomosing systems (Spiegelman et al., 2001; Hart, 1993). If such systems are still described 
diagram (Fig. 4) still is useful to provide first order estimates of thermal non-equilibrium temperature differences by identifying the regimes in which the parameters are expected to vary with depth. A more rigorous evaluation of thermal non-equilibrium temperatures for well-defined anastomosing systems with prescribed parameters $\phi(z), P e(z), A(z)$ will be presented in another paper (Chevalier and Schmeling, in prep.).

\subsection{Time scales}

It is interesting to evaluate the time scales for reaching the maximum non-equilibrium temperature differences and the steady state. For every model, we recorded the time needed to reach $90 \%$ of the maximum temperature differences between fluid and solid, $t_{90 \%}$, and the time needed to reach steady-state, $t_{\text {steady }}$. These times can be compared with different time scales that may characterize the evolution of temperatures in the models. These time scales can be based on advection over a characteristic distance $d_{c h a r}$ and read $t_{a d v d}=d_{c h a r} / v_{0}$, or on diffusion over the characteristic distance giving $t_{\text {diffd }}=d_{c h a r}^{2} /\left(c_{t h}^{2} \kappa_{0}\right)$, We tested these time scales with various length scales of the models, namely, the prescribed boundary layer thickness, $d m$, the geometric mean $\sqrt{\left(d m L_{0}\right)}$ which corresponds to the scaling time $t_{0}$, the channel width $L_{0}$, and the model height $H$.

Grouping the models depending on the regime (see section 4.3.6, and Fig. 4) they belong to, we plotted the recorded times $t_{90 \%}$ and the time to reach steady state versus the characteristic time scales mentioned above to evaluate which time scale fits best to the observed times. The result is shown in Figure 7.

- In regime 1 (high Pe), $t_{90 \%}$ is proportional to $t_{a d v H}$ (Figure 7a, circles). In this regime the high value of Pe makes the fluid temperature increase fast. It reaches its maximum value during the time under which significant fluid-solid heat transfer occurs, after traveling the full distance $H$. Depending on the value of $A$, which quantifies the efficiency of heat transfer, the temperature difference can then decrease before steady state is reached. The time for reaching steady state (Fig. 7b, circles) varies almost linearly with $t_{\text {steady }} \propto t_{\text {diffH }}$ but is up to one orders of magnitude larger. Clearly it is also controlled by diffusion. In this regime fluid temperature increases rapidly, then solid temperature increases, possibly leading to further fluid temperature increase, until heat transfer and diffusion equilibrate for the solid that reaches a steady state.

- In regime 2 (low $P e$ and low $A$ ) the time for reaching $\Delta T_{\max }$ is controlled by interfacial heat transfer (Fig. 7a, asterisks) on the length scale $\sqrt{\left(d m L_{0}\right)}$ as long as $t_{90 \%}$ is proportional to $t_{0}$, but for very small $A$ (higher $t_{0}$, equivalent to inefficient heat transfer) thermal non-equilibrium is reached earlier than $t_{0}$ as it is limited by diffusion through the whole domain which is probably the reason for flattening the $t_{90 \%}$ curve. The time for reaching steady state is controlled by the diffusion time scale (Fig. 7b), but after a somewhat longer time.

- In regime 3, $(P e / A<1, A>1)$, time for reaching $\Delta T_{\text {max }}$ depends mostly on $A$ (i.e. interfacial diffusion on the length scale $\left.\sqrt{\left(d m L_{0}\right)}\right)$. In this regime, heat transfer is no more negligible compared with advection, and limits the temperature difference that can develop. Steady state is reached at a time that is controlled by advection time scale (not shown), but limited by diffusion time scale (Fig. 7b, crosses). 


\subsection{Applications to magmatic systems}

We now test the possible occurrence of thermal non-equilibrium in natural magmatic systems based on the suggested controlling non-dimensional parameters, namely the Peclet number $P e$ and the heat transfer number $A$. Due to the smaller importance of $\phi$ (c.f. section 4.3.6) we neglect the influence of $\phi$ and focus on $P e$ and $A$ only. Typical melt flow stages for mid-ocean ridges include stage $a$ ), partially molten regions with interstitial melts sitting at grain corners, grain edges or grain faces with low (0.0001 - 6\%) melt fractions (see e.g. the discussion in Schmeling, 2006), stage $b$ ), merging melt channel or vein systems with high (> $10-20 \%$ ) porosity channels identified as dunite channels after complete melt extraction (Kelemen et al., 1997), and stage $c$ ), propagating dykes or other volcanic conduits. Schmeling et al. (2018) discussed possible Peclet numbers for such systems based on a Darcy flow based Peclet number

$P e_{D}=\frac{v_{D} d_{S}}{\kappa_{0}}$

which relates to the Peclet number used here by $P e=P e_{D} /(1-\phi)$ for melt in channels or $P e=P e_{D} /(\sqrt{\phi}(1-\sqrt{\phi}))$ for melt in tubes. Schmeling et al. (2018) estimated $P e_{D^{-}}$numbers for the three stages as $10^{-5}$ to $10^{-7}$ for stage $a$ ), $10^{-4}$ to $10^{-5}$ for stage $b$ ) at depths where channel distances are of order $0.1 \mathrm{~m}$, and $10^{-4}$ to 1 at shallower depths where the channel distances have increased to the order of $1 \mathrm{~m}$ to $1 \mathrm{~km}$, and $>10^{4}$ for the dyke stage $c$ ). Obviously, the Peclet number used here is of the same order as $P e_{D}$ for melt in tabular channels, but may be about one half order of magnitude larger than $P e_{D}$ for tubular melt conduits.

To estimate realistic ranges for $A$ typical interfacial thermal boundary layer thicknesses may be considered for the above mentioned stages. Following the arguments from section 5.1.4 a good estimate for the interfacial boundary layer thickness is $d m=c_{t h} \sqrt{\kappa_{0} t}$ : Assuming that the characteristic time can be expressed by the fluid velocity $v_{0}$ and system height $H$, i.e. by $t=H / v_{0}$, we may express $v_{0}$ in terms of the Peclet number. With the resulting $t$ and subsequent $d m$ we obtain a scaling law for $A$ :

$A=\sqrt{P e} \sqrt{\frac{L_{0}}{H}}$

475 For mid ocean ridge settings we assume $H$ of the order 1 to $10 \mathrm{~km}$ and $L_{0}$ increasing from $10^{-5} \mathrm{~m}$ (stage $a$ ), interstitial melts) to $10^{-2} \mathrm{~m}$ to $10^{2} \mathrm{~m}$ for the channeling stage $b$ ) (see Schmeling et al., 2018) to $>10 \mathrm{~m}$ for the dyke stage $c$ ). With these estimates the above scaling law (Eq. 32) allows estimating $A$ of order $10^{-8}$ to $10^{-6}$ for stage $a$ ), order $10^{-5}$ in the early phase and order $10^{-}$ 4.5 to $10^{-0.5}$ in the later phase appropriate for dunite systems for stage $b$ ), and order 10 for the dyke stage $c$ ).

These resulting stages for $P e$ and $A$ are indicated in Figure 4. Starting from interstitial melts at full thermal equilibrium, channeling and veining may result in moderate thermal non-equilibrium, while after transition to dyking full thermal nonequilibrium is predicted. 
A similar exercise could be made for continental magmatic systems. We skip such an explicit evaluation here but note that silicic melt viscosities are typically higher than those of basaltic melts at mid-ocean ridges. Thus, Peclet numbers and heat transfer numbers are expected to be smaller, resulting in a downward and leftward shift of the natural stages in Figure 4.

\section{Conclusions}

In conclusion we showed that in magmatic systems characterized by two-phase flows of melts with respect to solid, thermalnon-equilibrium between melt and solid may arise and become important under certain conditions. The main conclusions are summarized as follows:

From non-dimensionalization of the governing equations three non-dimensional numbers can be identified controlling thermal non-equilibrium: the Peclet number $P e$, the heat transfer number $A$, and the melt porosity $\phi$. Both numerical and analytical solutions show that in a $P e-A$ - parameter space three regimes can be identified:

- In regime 1 (high Pe $(>1)$ ) strong thermal non-equilibrium develops independently of Pe and A, a scaling law $T_{f}-$ $T_{S}=\frac{\Delta T}{H} z$ has been derived.

- In regime 2 (low Pe $(<1)$ and low A $(<1)$ ) non-equilibrium decreases proportional to decreasing Pe, the scaling law reads $T_{f}-T_{s}=\operatorname{Pe} \Delta \operatorname{Tz}\left(1-\frac{z}{2 H}\right)$.

- In regime 3 (low Pe $(<1)$ and large A $(>1)$ ) non-equilbriums scales with Pe/A and thus becomes unimportant, the scaling law is $T_{f}-T_{s}=(1-\phi) \frac{P e}{A} \frac{\Delta T}{H}$.

Further conclusions include:

- The melt porosity $\phi$ has only a minor effect on thermal non-equilibrium.

- The time scales for reaching thermal non-equilibrium scale with the advective time-scale in the high $P e$-regime and with the interfacial diffusion time in the other two low Pe number regimes.

- Applying the results to natural magmatic systems such as mid-ocean ridges can be done by estimating appropriate orders of $P e$ and $A$. Plotting such typical ranges in the $P e-A$ regime diagram reveals that a) interstitial melt flow is in thermal equilibrium, b) melt channeling as e.g. revealed by dunite channels may reach moderate thermal nonequilibrium, and c) the dyke regime is at full thermal non-equilibrium.

While for simplicity the presented approach has been done essentially for constant model parameters, it can easily be extended to vertically varying parameters. Thus, tools are provided for evaluating the transition from thermal equilibrium to nonequilibrium for anastomosing systems (Hart, 1993; Chevalier and Schmeling, in prep.).

\section{Acknowledgements}

510 We acknowledge funding support by the Deutsche Forschungsgemeinschaft (DFG) with the grant no. 403710316. 
https://doi.org/10.5194/se-2021-149

Preprint. Discussion started: 21 December 2021

(c) Author(s) 2021. CC BY 4.0 License.

(c) (i)

\section{References}

Aharonov, E., Whitehead, J.A., Kelemen, P. B., and Spiegelman, M.: Channeling instability of upwelling melt in the mantle. J. Geophys. Res., 100, 20,433 - 20,450, 1995.

Amiri, A. and Vafai, K.: Analysis of Dispersion Effects and Non-Thermal Equilibrium, Non-Darcian, Variable Porosity Incompressible Flow Through Porous Media, Int. J. Heat Mass Transf., 37, 939-954, 1994.

Becker, K. and Davis, E.: On situ determinations of the permeability of the igneaous oceanic crust, in: Hydrogeology of the Oceanic Lithosphere, pp. 311-336, eds. Davis, E. \& Elderfield, H., CambridgeUniv. Press, 2004.

Bruce, P. M. and Huppert, H. E.: Solidification and melting along dykes by the laminar flow of basaltic magma. In Ryan, M. P., ed., Magma transport and storage. Chichester: Wiley, pp. 87-101, 1990.

Davis, E.E. et al.: Regional heat flow variations across the sedimented Juan de Fuca ridge eastern flank: constraints on lithospheric cooling and lateral hydrothermal heat transport, J. geophys. Res., 104, 17 675-17 688, 1999.

de Lemos, M. J. S.: Thermal non-equilibrium in heterogeneous media, Springer Science+Business Media, Inc., 2016.

Furbish, D. J.: Fluid Physics in geology. Oxford University Press, New York. 476 pp., 1997.

Harris, R.N. and Chapman, D.S.: Deep seated oceanic heat flow, heat deficits and hydrothermal circulation, in Hydrogeology

525 of the Oceanic Lithosphere, pp. 311-336, eds Davis, E. \& Elderfield, H., CambridgeUniv. Press, 2004.

Hart, S.R.: Equilibration during mantle melting: a fractal tree model. Proc. Natl. Acad. Sci. USA 90, 11,914-11,918, 1993.

Kelemen, P. B., Whitehead, J. A., Aharonov, E., and Jordahl, K. A.: Experiments on flow focusing in soluble porous media, with applications to melt extraction from the mantle, J. Geophys. Res. 100, 475 - 496. doi.org/10.1029/94JB02544, 1995.

Kelemen, P. B., Hirth, G., Shimizu, N., Spiegelman, M., and Dick, H. J. B.: A review of melt migration processes in the adiabatically upwelling mantle beneath oceanic spreading ridges. Phil. Trans. R. Soc. Lond. A, 355, 283 - 318, 1997.

Keller, T., May, D. A., and Kaus, B. J. P.: Numerical modelling of magma dynamics coupled to tectonic deformation of lithosphere and crust, Geophys. J. Int., 195.3, 1406-1442, 2013.

Landwehr, D., Blundy, J., Chamorro-Perez , E. M., Hill, E., and Wood, B.: U-series disequilibria generated by partial melting of spinel lherzolite, Earth Planet. Sci. Lett., 188 329-348, 2001.

535 Lister, J. R. and Kerr, R. C.: Fluid-mechanical models of crack propagation and their application to magma transport in dykes. J. Geophys. Res. 96, 10049 - 10 077, 1991.

Maccaferri, F., Bonafede, M., Rivalta, E.: A quantitative study of the mechanisms governing dike propagation, dike arrest and sill formation. J. Volc. Geoth. Res. 208, 39-50, 2011.

McKenzie, D.: The generation and compaction of partially molten rock. J. Petr., 25, 713-765, 1984.

540 McKenzie, D.: Constraints on melt generation and transport from U-series activity ratios. Chem. Geol., 162, 81 - 94, 2000.

Minkowycz, W. J., Haji-Sheikh, A., Vafai, K.: On departure from local thermal equilibrium in porous media due to a rapidly changing heat source: the Sparrow number, Int. J. Heat Mass Transf., 42 (18), 3373-3385, 1999.

Nield, D. A. and Bejan, A.: Convection in Porous Media, Third Edition, Springer Science+Business Media, Inc., 2006. 
https://doi.org/10.5194/se-2021-149

Preprint. Discussion started: 21 December 2021

(c) Author(s) 2021. CC BY 4.0 License.

(c) (1)

Rivalta, E., Taisne, B., Buger, A. P., and Katz, R. F.: A review of mechanical models of dike propagation: Schools of thought, 545 results and future directions, Tectonophysics, 638, $1-42,2015$.

Roy, M.: Thermal disequilibrium during melt-transport: Implications for the evolution of the lithosphere-asthenosphere boundary. arXiv preprint arXiv:2009.01496, 2020.

Rubin, A. M.: Propagation of magma-filled cracks. Annual Review of Earth and Planetary Sciences 23, 287-336, 1995.

Schmeling, H.: A model of episodic melt extraction for plumes, J. Geophys. Res., 111, B03202, doi:10.1029/2004JB003423, 5502006.

Schmeling, H, Marquart, G, and Grebe, M.: A porous flow approach to model thermal non-equilibrium applicable to melt migration, Geophys. J. Int., 212, 119-138, 2018.

Spiegelmann, M., Kelemen, P. B., Aharonov, E.: Causes and consequences of flow organization during melt transport: The reaction infiltration instability in compactible media. J. Geophys. Res., 106, 2061 - 2077, 2001.

555 Turcotte, D. and Schubert, G.: Geodynamics. Cambridge University Press,Cambridge, 2014.

Verruijt, A.: Theory of Groundwater Flow. The Macmillan Press Ltd., London and Basingstoke. 141 pp. DOI 10.1007/978-1349-16769-2, 1982.

Wilcock, W. S. D. and Fisher, A. T.: Geophysical constraints on the subseafloor environment near Mid-Ocean ridges, pp. 51

- 74, in: Subseafloor Biosphere, eds. C. Cary, E. Delong, D. Kelley, and W. S. D. Wilcock. Washington DC. American 560 Geophysical Union, 2004.

Woods, A. W.: Flow in Porous Rocks: Energy and Environmental Applications. Cambridge University Press, Cambridge, 289 pp., 2015. 


Symbol Definition Units

\begin{tabular}{|c|c|c|}
\hline$A$ & Heat transfer number, Eq. $(10 a, b)$ & - \\
\hline$c_{p, f, s, 0}$ & $\begin{array}{l}\text { Specific heat at constant pressure for the fluid, solid, or reference, } \\
\text { respectively }\end{array}$ & $\mathrm{J} \mathrm{kg}^{-1} \mathrm{~K}^{-1}$ \\
\hline$c$ & Geometrical constant, 2 for channels, 4 for tubes (Eq. 9b, 10b) & - \\
\hline$c_{t h}$ & Constant for thermal boundary layer, 2.32 for cooling half space & - \\
\hline$d m$ & Interfacial boundary layer thickness & $\mathrm{m}$ \\
\hline$d_{s}, d_{f}$ & Characteristic length scale of solid or fluid phase, respectively & $\mathrm{m}$ \\
\hline$f$ & Subscript used for fluid & - \\
\hline$H$ & Height of the model & $\mathrm{m}$ \\
\hline$L_{0}$ & Scaling length used for non-dimensionalization $\left(=d_{f}\right)$ & $\mathrm{m}$ \\
\hline$P e$ & Peclet number, Eq. (14) & - \\
\hline$Q_{f s}$ & Interfacial heat exchange rate from fluid to solid & $\mathrm{J} \mathrm{s}^{-1} \mathrm{~m}^{-3}$ \\
\hline$S$ & Subscript used for solid & - \\
\hline$S$ & Interfacial area density, i.e. interfacial area per volume & $\mathrm{m}^{-1}$ \\
\hline$t$ & Time & $\mathrm{s}$ \\
\hline$t_{0}$ & Diffusion time on interfacial scale used for non-dimensionalization & $\mathrm{s}$ \\
\hline$T_{f, s}$ & Temperature of the fluid or solid, respectively & $\mathrm{K}$ \\
\hline
\end{tabular}


$\Delta T_{0} \quad$ Initial temperature difference between top and bottom

$v_{f, s} \quad$ Velocity of the fluid or solid, respectively

$v_{0} \quad$ Constant fluid velocity in the model

$v_{D} \quad$ Volmetric flow rate (Darcy velocity) $\left(=\phi v_{0}\right)$

$x, y, z$

$\kappa_{f, s, 0}$

$\lambda_{f, s}$

$\lambda_{\text {eff }}$

$\phi, \phi_{0}$

$\rho_{f, s, 0}$
Coordinates, distance

Thermal diffusivity of the fluid, solid or reference, respectively

Thermal conductivity of the fluid or solid, respectively

Effective thermal conductivity at the solid-fluid interface

Porosity or scaling porosity, respectively

Density of the fluid, solid, or reference. respectively
K

$\mathrm{m} \mathrm{s}^{-1}$

$\mathrm{m} \mathrm{s}^{-1}$

$\mathrm{m} \mathrm{s}^{-1}$

$\mathrm{m}$

$\mathrm{m}^{2} \mathrm{~s}^{-1}$

$\mathrm{W} \mathrm{m} \mathrm{m}^{-1} \mathrm{~K}^{-1}$

$\mathrm{W} \mathrm{m} \mathrm{m}^{-1} \mathrm{~K}^{-1}$

$\mathrm{kg} \mathrm{m}^{-3}$

Table 1: Symbols, their definition, and physical units used in this study. 

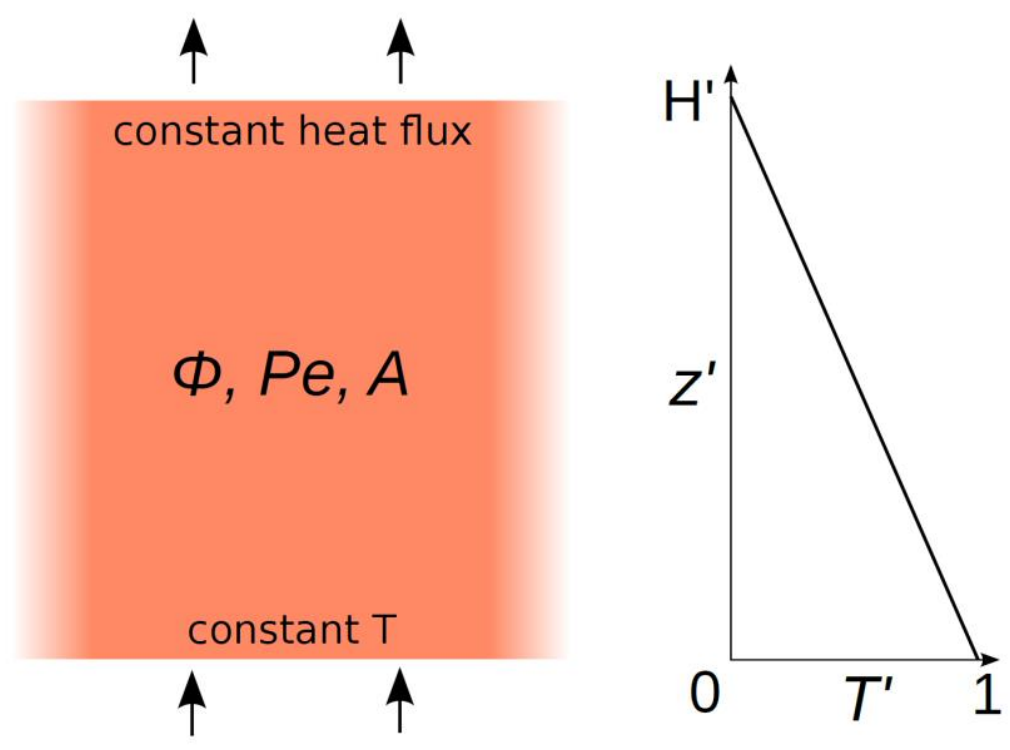

Figure 1. Initial and boundary conditions. 
https://doi.org/10.5194/se-2021-149

Preprint. Discussion started: 21 December 2021

(c) Author(s) 2021. CC BY 4.0 License.
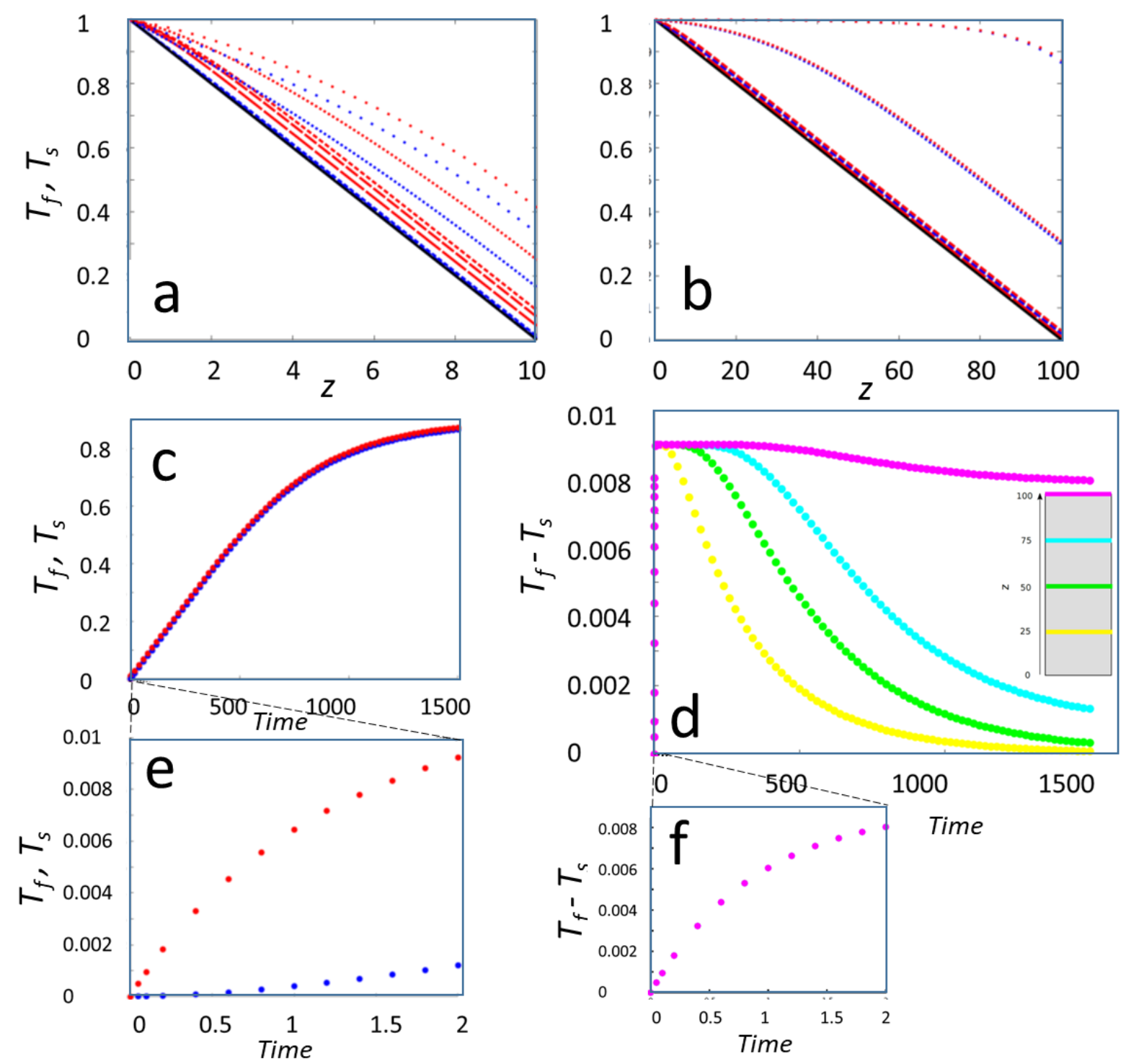

Figure 2. Typical model evolution for $P e=1, A=1, \phi=0.1$. a) Model 1 with non-dimensional height $H=10$. Red and blue curves show the fluid and solid temperatures at different times, respectively. Initial temperatures are in black. b) $M o d e l ~ 2$ with $H=100$, else as in a). c) Temporal evolution of fluid and solid temperatures, $T_{f}$ (red) and $T_{s}$ (blue), respectively, of model 2 at the top. d) Evolution of fluid - solid temperature difference $\left(T_{f}-T_{s}\right)$ at different distances $z$ in model 2. The positions $z=25,50,75$, and 100 are indicated by the inset. e) Zoomed-in early temporal evolution of model shown in c). f) Zoomed-in early temporal evolution of model shown in d). 
https://doi.org/10.5194/se-2021-149

Preprint. Discussion started: 21 December 2021

(c) Author(s) 2021. CC BY 4.0 License.
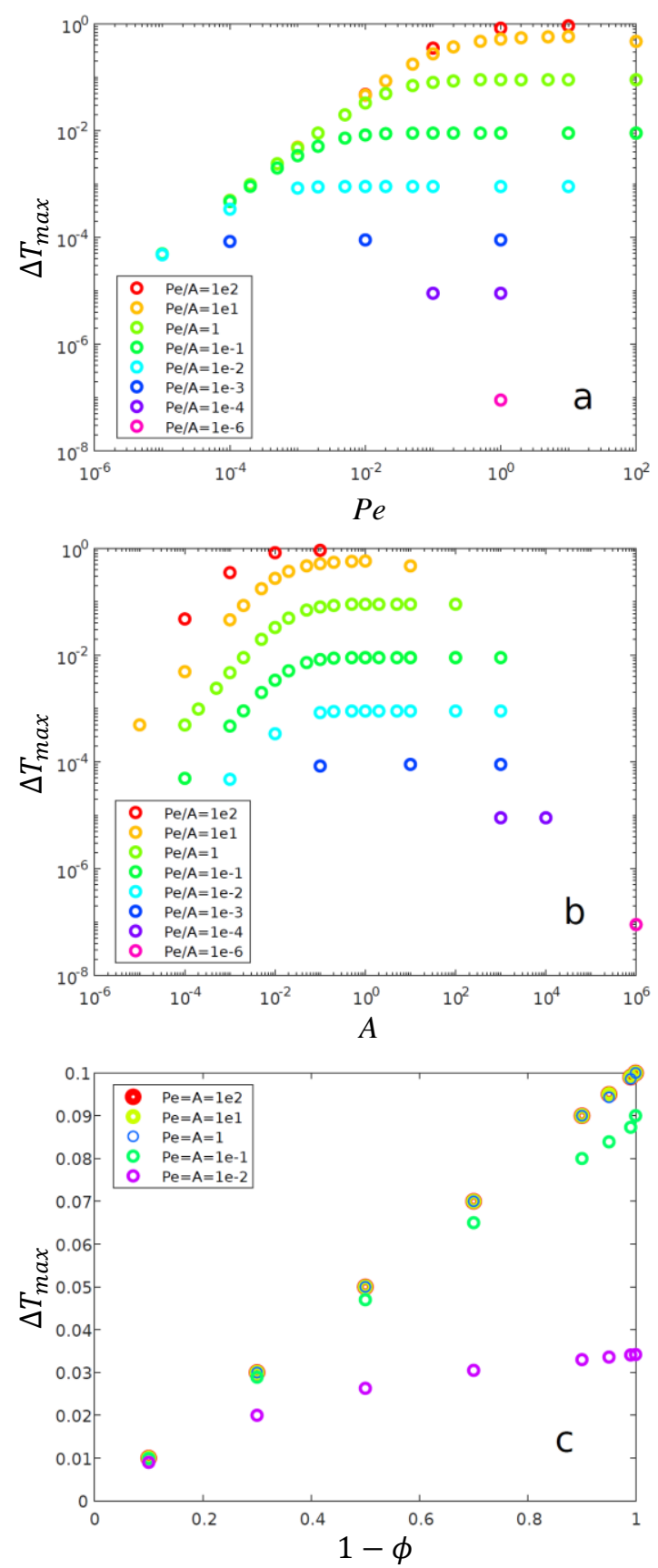

Figure 3. Maximum fluid - solid temperature differences $\Delta T_{\text {max }}$ of numerical models with different parameters, plotted as a function of a) the Peclet number Pe, b) the heat transfer number $A$, and c) the solid fraction $(1-\phi)$. 


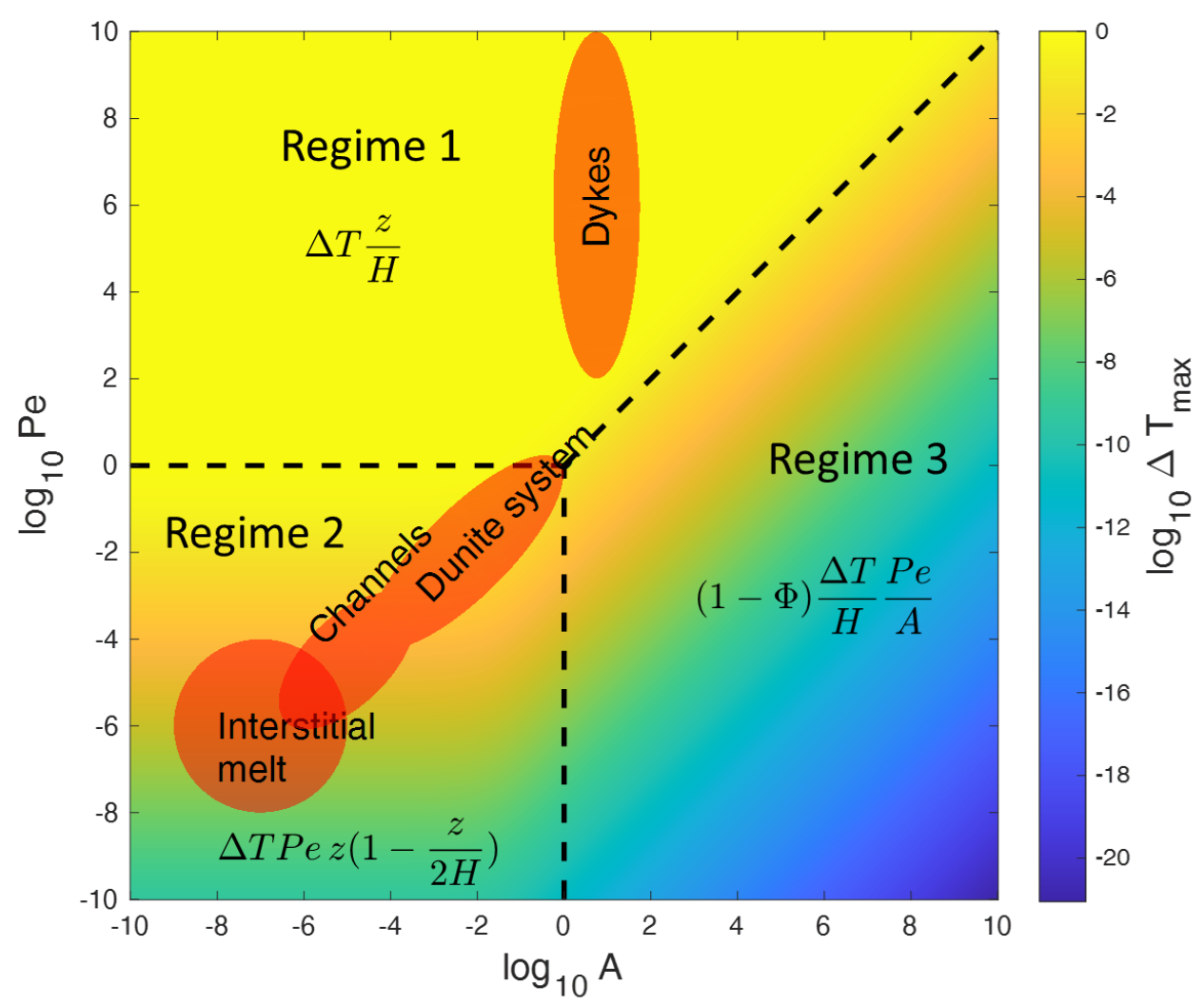

Figure 4. Main regimes of the maximum fluid - solid temperature differences $\Delta T_{\max }$ due to thermal non-equilibrium obtained by the analytical solution (equ. 20) and associated limits in the parameter space of the heat transfer number $A$ and the Peclet number Pe. The melt fraction $\phi$ has been assumed as 0.1. The asymptotic limits are indicated by the formulas. Regime boundaries are shown as dashed lines. Typical parameter combinations for magmatic settings such as interstitial melts or dykes are indicated by the orange ellipses. 
https://doi.org/10.5194/se-2021-149

Preprint. Discussion started: 21 December 2021

(c) Author(s) 2021. CC BY 4.0 License.
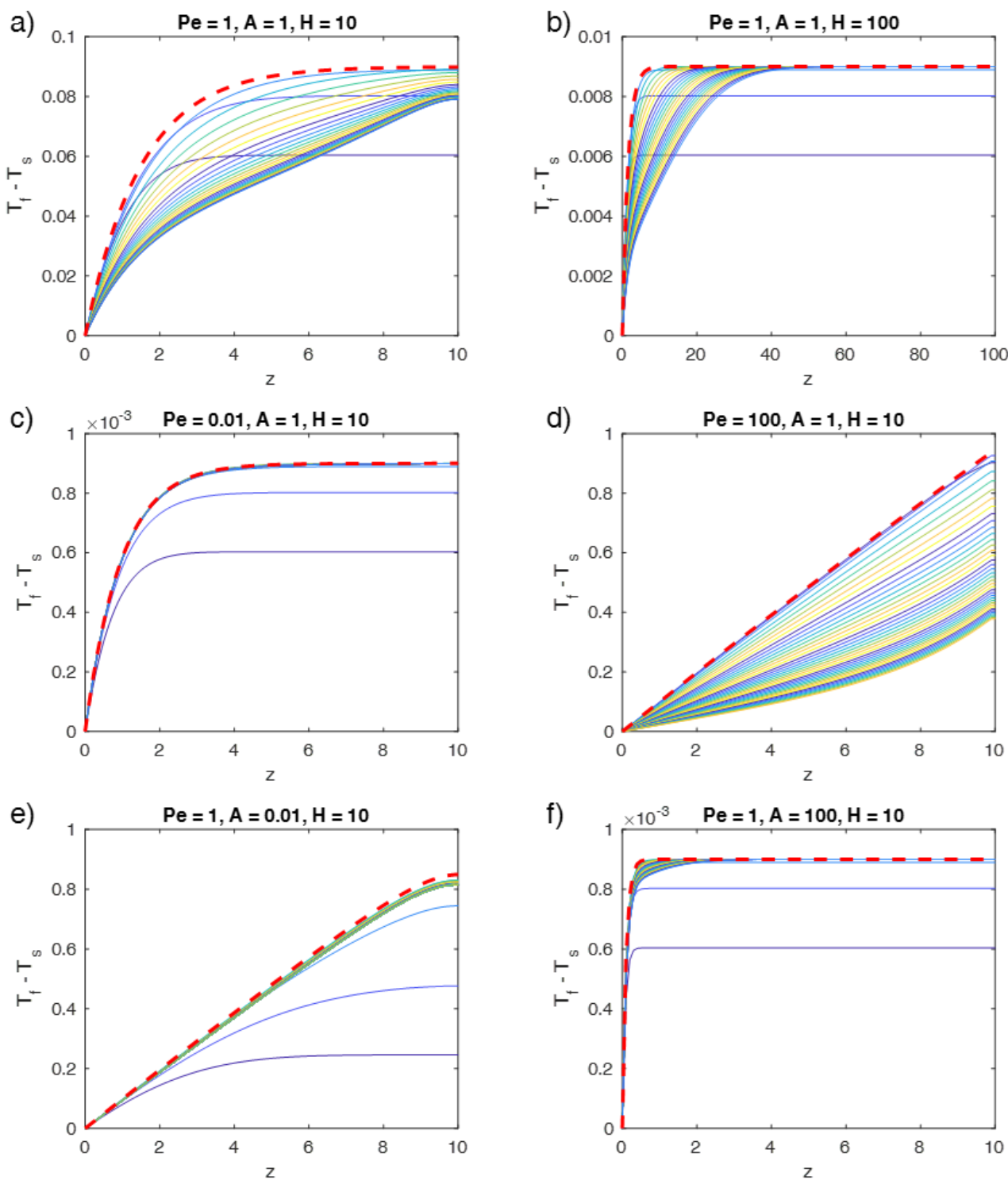

Figure 5. Comparison of depth- and time- dependent numerical solutions with the time - independent analytical solutions for different parameters $\mathrm{Pe}, \mathrm{A}$, and $\mathrm{H}$ as indicated in the sub-figure titles. The thin curves in each panel show $\left(T_{f}-T_{s}\right)-$ profiles for progressive times, the colors are cyclically varied with time from blue to yellow, starting with blue. The bold red curve shows the analytical solution equ. (20), which represents a very good estimate of the depth-dependent temporal maximum of the temperature difference. The total non-dimensional times of each panel are: a) - c): 100, d) 15, e) 10, and f) 100 . As porosity $\phi=0.1$ is assumed. 
https://doi.org/10.5194/se-2021-149

Preprint. Discussion started: 21 December 2021

(c) Author(s) 2021. CC BY 4.0 License.
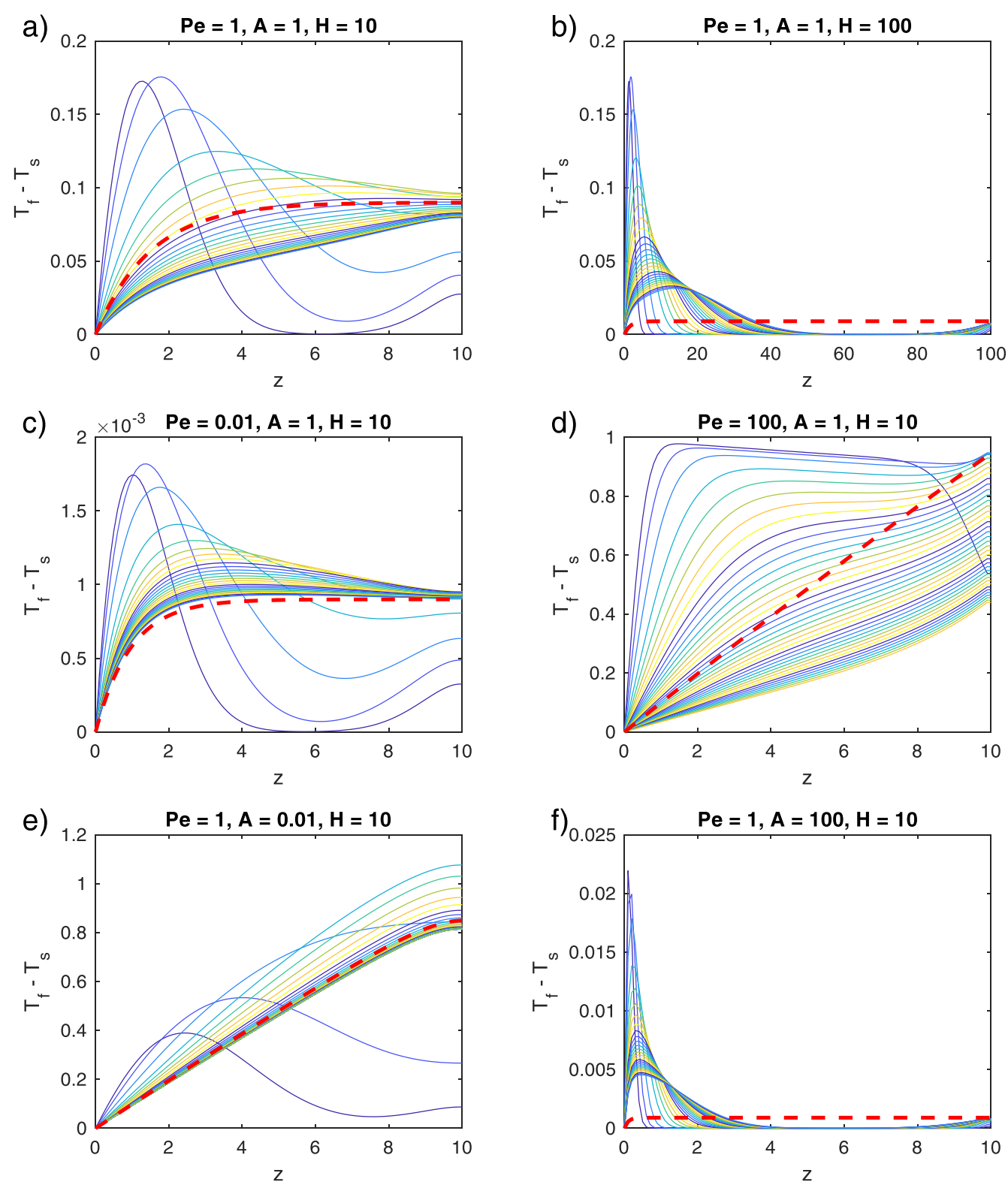

610 Figure 6. Time- and depth- dependent numerical solutions (thin curves) as in Figure 5 but for step-function initial conditions: $\boldsymbol{T}_{\boldsymbol{f}}=$ $T_{s}=1$ at $\mathrm{z}=0$ and $T_{f}=T_{s}=0$ at $\mathrm{z}>0$ at $\mathrm{t}=0$. Dashed curves are the time-independent analytical solutions as in Fig. 5. 

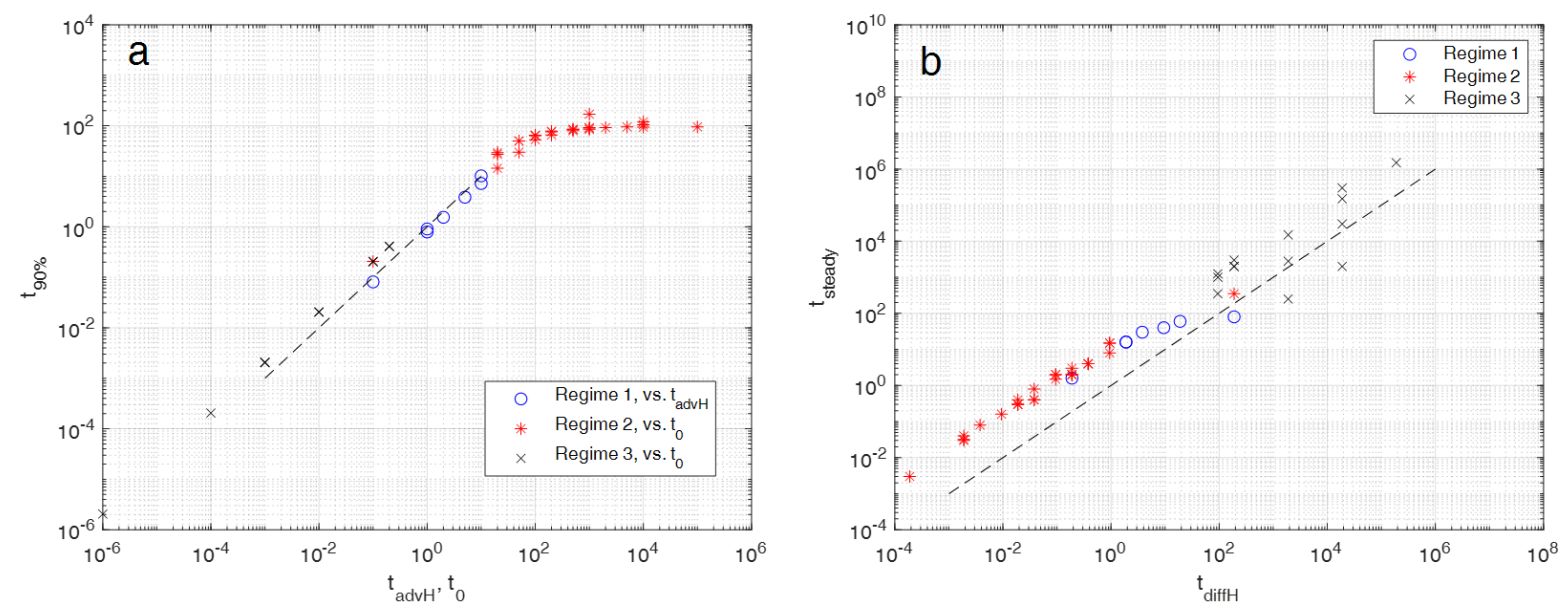

Figure 7. For evaluating time scales the numerically determined times of models with various parameters $\mathrm{Pe}, \mathrm{A}$ representing the three different regimes 1, 2 and 3 (different symbols) are plotted against characteristic scaling times. a) times for reaching $90 \%$ of the maximum temperature difference $\Delta \boldsymbol{T}_{\max }$ are plotted against either the advective time scale tadvH based on model height $\mathrm{H}$ for regime 1 models or against the to time scale for regime 2 and 3 models. b) times for reaching steady states are plotted against the characteristic diffusive time scales, tdiffH, based on model height $\mathbf{H}$ for all 3 regimes. Models close to the dashed line $(y=x)$ are in best agreement with the characteristic times. In this Figure the times were taken dimensional by setting LO and $\kappa_{0}$ to 1 to allow for various to's. 